\title{
Field trial of antibody response to inactivated bacterial vaccine in young Holstein calves: influence of animal health status
}

\author{
Kazusa Mori ${ }^{1}$, Konosuke Otomaru ${ }^{2}$, Toshihide Kato ${ }^{1}$, Osamu Yokota ${ }^{3}$, Hiromichi Ohtsuka ${ }^{1 凶}$ \\ ${ }^{1}$ School of Veterinary Medicine, Rakuno Gakuen University, Ebetsu, Hokkaido 069-8501, Japan \\ ${ }^{2}$ Joint Faculty of Veterinary Medicine, Kagoshima University, Kagoshima 890-0065, Japan \\ ${ }^{3}$ F. SIDE veterinary service Sapporo, Hokkaido 004-0072, Japan \\ ohtsuka@rakuno.ac.jp
}

Received: July 4, $2021 \quad$ Accepted: January 11, 2022

\begin{abstract}
Introduction: Bovine respiratory disease (BRD) is one of the primary causes of death in young calves. Vaccination against infection by the common bacteria causing BRD is possible; however, the physical condition of the young calves that enables antibody production when stimulated by early immunisation remains to be elucidated. Material and Methods: Healthy young female Holstein calves on a commercial dairy farm were fed a colostrum replacer and administered primary and booster immunisations with an inactivated vaccine against the bacterial pneumonia agents Histophilus somni, Pasteurella multocida and Mannheimia haemolytica. At each immunisation, the body weight and height at the withers were measured and the body mass index (BMI) was calculated. Blood was sampled immediately before immunisation and 3 weeks following the booster. The calves were divided into positive and negative groups based on the antibody titre at the final blood sampling. Maternal antibody titres at the primary immunisation and BMI, nutritional status and oxidative stress at both immunisations were compared between the two groups. Results: Antibody titre at the primary and BMI at both immunisations were significantly higher in the positive than in the negative group $(\mathrm{P}<0.05)$. Additionally, serum gamma globulin was significantly higher in the positive group $(\mathrm{P}<0.05)$, indicating a strong correlation between maternal antibody and serum gamma globulin levels. Conclusion: Elevated maternal antibody titre and higher BMI are positive factors for successful early immunisation, for which suitable colostrum may also be fundamental in young calves administered inactivated vaccines.
\end{abstract}

Keywords: early vaccination, bovine respiratory disease, maternal antibody, nutritional status, oxidative stress.

\section{Introduction}

Bovine respiratory disease (BRD) is one of the primary causes of death in pre-weaned calves and weaned dairy heifers (28). It is a multifactorial disease; therefore, development of effective control strategies has been difficult to accomplish. Immunisation against bovine respiratory bacteria is a useful tool for reducing the risk of this disease (27). A previous study involving Holstein calves which were initially immunised at 7 days of life or later reported that administration of a booster immunisation with a vaccine containing inactivated antigens of the bacteria causing pneumonia resulted in increased antibody titres. However, the conditions for young immunologically naïve calves (11) to produce antibodies by immunisation stimulation still remain to be elucidated (15).
The health of young calves is closely associated with passive immunity (6). Despite this, it has been proposed that maternal antibodies inhibit the response to immunisation (7). Hodgins et al. (9) reported that Holstein dairy calves immunised at 2 and 4 weeks of life with live Mannheimia haemolytica vaccine show weaker antibody responses than calves immunised at 6 and 8 weeks. In contrast, our previous study suggested that after the administration of an initial and booster immunisation with a vaccine containing inactivated antigens of Histophilus somni, Pasteurella multocida and Mannheimia haemolytica, the titres of young calves with high maternal antibody levels (MAL) were not lower than those of calves with low MAL. In addition, the percentage of calves that became seropositive was higher in the group with high MAL than in the group with low MAL (15). Whether maternal antibodies 
inhibit the effectiveness of inactivated vaccines is still unclear.

The health of a calf is associated with body weight, nutritional status and oxidative stress (OS) $(17,18,24)$. It has been noted that immune function is reduced in suckling calves with low body weights or in those given insufficient nutrition $(17,18)$. Additionally, OS diminishes the functional capability of immune cells, increases susceptibility to diseases (24) and has been implicated in multiple disease processes, including respiratory and neonatal diseases (5). Therefore, OS might play a significant role in the health of the neonatal calf (2). For these reasons, it has been suggested that underweight, malnutrition and OS may be associated with the response to immunisation in young calves. However, few reports are available that describe the relationship between body weight, nutritional status and OS and response to immunisation in young calves.

In this report we describe the relationship between a calf's MAL, body weight, nutritional status and serum OS and that animal's antibody response to early immunisation with an inactivated bacterial vaccine against BRD bacteria. The main objective of this study was to elucidate the necessary conditions for young Holstein calves to produce antibodies.

\section{Material and Methods}

This was a case-control study that included 107 healthy female Holstein calves on a commercial dairy farm. All calves were born on the farm. Each was fed once two bags of $200 \mathrm{~g}$ of colostrum replacer (Calfsupport Dash, Zenoaq, Koriyama, Japan) in $2 \mathrm{~L}$ of water at approximately $50^{\circ} \mathrm{C}$. The vaccine contained inactivated antigens of $H$. somni, P. multocida and M. haemolytica (Cattlebact 3 combo, strain no. 26-1, Kyoto Biken Laboratories, Uji, Japan). Following the grant of the owner's informed consent, all calves received $1 \mathrm{~mL}$ of a primary intramuscular immunisation between the ages of 1 and 3 weeks and a booster immunisation three weeks later. We have previously reported that little difference in antibody titres was observed following the initial immunisation at 1, 2 or 3 weeks of life and the booster (15).

In the first experiment, the calves body weights and withers heights were measured on the day of each immunisation. Body mass index (BMI), widely used as a measure of underweight or normal weight in humans, was calculated by dividing the body weight by the square of the height in metres (29). Blood was drawn immediately prior to each immunisation, as well as three weeks subsequent to the booster immunisation. Blood samples were collected into plain vacutainer tubes and centrifuged at 3,500 rpm for $8 \mathrm{~min}$, and the serum was isolated. Serum was maintained at $-30^{\circ} \mathrm{C}$ until analysis and serum antibodies against $H$. somni, $P$. multocida and $M$. haemolytica were detected using an ELISA as previously described (16). When the antibody titres for P. multocida and $M$. haemolytica were detected with less than a 100 -fold dilution, they were calculated as a 50-fold dilution.
In the second experiment, in addition to antibody titres and BMI, calves had total cholesterol (T-cho), blood urea nitrogen (BUN), total protein, albumin/ globulin $(\mathrm{A} / \mathrm{G})$ ratio, albumin, alpha globulin, beta globulin and gamma globulin measured to investigate their nutritional status. These laboratory studies were conducted by the Daiichi Kishimoto Rinsho Kensa Center and involved 66 of the 107 calves.

In the third experiment, 47 of the 66 calves evaluated in the second experiment were additionally investigated for OS. Serum-free radicals were determined by the concentration of diacron reactive oxygen metabolites (d-ROMs). The serum d-ROMs test was expressed as arbitrary Carratelli units (Carr U). One Carr U corresponds to $0.8 \mathrm{mg} / \mathrm{L}$ hydrogen peroxide (1). Serum antioxidant capacity was determined as the concentration of biological antioxidant potential (BAP), a global measurement of many antioxidants (4). The methods used to measure OS were described previously by Otomaru et al. (21). The oxidative stress index (OSI) was calculated from the d-ROMs and BAP results using the ratio of d-ROMs to BAP multiplied by 100 (1).

In each experiment, antibody titres greater than 0.604 (H. somni), 100 (P. multocida) and 200 (M. haemolytica) were considered to be positive $(15,20)$. Based on the titres 3 weeks subsequent to the booster immunisation, calves were divided into positive and negative groups for H. somni, P. multocida or M. haemolytica. Maternal antibody titres at the initial immunisation and BMI, nutritional status and OS at each immunisation were compared between the positive and negative calves for each of the bacteria. To investigate the conferral of protection by maternal antibodies, the number of calves positive and negative for $P$. multocida and $M$. haemolytica antibodies was compared for each MAL in the first experiment.

Data were expressed as geometric mean $\pm \mathrm{SE}$ or as a scatter diagram. All analyses were conducted using XLSTAT 2020.1.1 (Addinsoft, Paris, France). The differences between positive and negative calves were examined using the Kolmogorov-Smirnov test. Fisher's exact test of independence was calculated to compare the number of positive and negative calves for each MAL. Pearson's correlation analyses were performed to assess the relationship between maternal antibody titre at the primary immunisation, BMI at both immunisations and antibody titre after the immunisations (first experiment) or between the maternal antibody titre and the calves' nutritional status at the primary immunisation (second experiment). A P value $<0.05$ was considered statistically significant.

\section{Results}

Table 1 presents the number of positive and negative calves for each of the three bacteria following immunisation. For P. multocida and M. haemolytica, the relationship between MAL and the number of positive and negative calves in the first experiment is also shown. No significant interaction between MAL and the number 
of positive or negative calves was found for $P$. multocida $(\mathrm{P}=0.421)$, but one interaction was found for $M$. haemolytica $(\mathrm{P}<0.01)$. In calves with high MAL against $M$. haemolytica (over 200), the number of $M$. haemolytica-positive calves was significantly higher than the number of negative calves (Table 1).

Table 1. The number of positive and negative calves following immunisation (a) in each experiment and (b) for each MAL in the first ex periment

\begin{tabular}{|c|c|c|c|c|c|c|c|}
\hline (a) & Experiment & Antibody titre & BMI & $\begin{array}{c}\text { Nutritional } \\
\text { status }\end{array}$ & $\begin{array}{c}\text { Oxidative } \\
\text { stress }\end{array}$ & Positive & Negative \\
\hline \multirow{3}{*}{ H. somni } & First & $\checkmark$ & $\checkmark$ & & & 77 & 30 \\
\hline & Second & $\checkmark$ & $\checkmark$ & $\checkmark$ & & 41 & 25 \\
\hline & Third & $\checkmark$ & $\checkmark$ & $\checkmark$ & $\checkmark$ & 25 & 22 \\
\hline \multirow{3}{*}{ P. multocida } & First & $\checkmark$ & $\checkmark$ & & & 101 & 6 \\
\hline & Second & $\checkmark$ & $\checkmark$ & $\checkmark$ & & 61 & 5 \\
\hline & Third & $\checkmark$ & $\checkmark$ & $\checkmark$ & $\checkmark$ & 42 & 5 \\
\hline \multirow{3}{*}{ M. haemolytica } & First & $\checkmark$ & $\checkmark$ & & & 37 & 70 \\
\hline & Second & $\checkmark$ & $\checkmark$ & $\checkmark$ & & 8 & 58 \\
\hline & Third & $\checkmark$ & $\checkmark$ & $\checkmark$ & $\checkmark$ & 8 & 39 \\
\hline (b) & & MAL & \multicolumn{2}{|c|}{ Positive } & Negative & \multicolumn{2}{|r|}{$P$ value } \\
\hline \multirow{4}{*}{ P. multocida } & & 50 & \multicolumn{2}{|c|}{38} & 4 & \multirow{4}{*}{\multicolumn{2}{|c|}{-}} \\
\hline & & 100 & \multicolumn{2}{|c|}{$\begin{array}{l}45 \\
15\end{array}$} & 1 & & \\
\hline & & 200 & & & 1 & & \\
\hline & & 400 & \multicolumn{2}{|c|}{3} & 0 & & \\
\hline \multirow{5}{*}{ M. haemolytica** } & & 50 & \multicolumn{2}{|c|}{3} & 16 & \multicolumn{2}{|r|}{0.058} \\
\hline & & 100 & \multicolumn{2}{|c|}{9} & 28 & \multicolumn{2}{|r|}{0.105} \\
\hline & & 200 & \multicolumn{2}{|c|}{12} & 19 & \multicolumn{2}{|r|}{0.566} \\
\hline & & 400 & \multicolumn{2}{|c|}{11} & 7 & \multicolumn{2}{|r|}{$0.009 * *$} \\
\hline & & 800 & \multicolumn{2}{|c|}{2} & 0 & \multicolumn{2}{|r|}{$0.050 *$} \\
\hline
\end{tabular}

No significant interaction between MAL and the number of positive or negative calves was found for Pasteurella multocida $(\mathrm{P}=0.421)$, but one was found for Mannheimia haemolytica $(\mathrm{P}<0.01)$. H. somni - Histophilus somni; $\mathrm{MAL}-$ maternal antibody level; * $-\mathrm{P}<0.05 ; * *-\mathrm{P}<0.01$

Table 2. The nutritional status in the second experiment (a) and oxidative stress marker values in the third experiment (b)

\begin{tabular}{|c|c|c|c|c|c|c|c|}
\hline \multirow{2}{*}{ (a) } & & \multicolumn{2}{|c|}{ H. somni } & \multicolumn{2}{|c|}{ P. multocida } & \multicolumn{2}{|c|}{ M. haemolytica } \\
\hline & & Positive & Negative & Positive & Negative & Positive & Negative \\
\hline \multirow{2}{*}{$\begin{array}{l}\text { Total cholesterol } \\
(\mathrm{mg} / \mathrm{dL})\end{array}$} & $\mathrm{P}$ & $93.6 \pm 5.3$ & $98.5 \pm 5.1$ & $96.1 \pm 4.0$ & $88.2 \pm 13.4$ & $79.3 \pm 8.3$ & $97.7 \pm 4.1$ \\
\hline & B & $118.3 \pm 5.2$ & $118.6 \pm 4.6$ & $118.7 \pm 3.8$ & $114.4 \pm 12.8$ & $113.8 \pm 10.0$ & $119.0 \pm 3.9$ \\
\hline \multirow{2}{*}{$\begin{array}{l}\text { Blood urea nitrogen } \\
(\mathrm{mg} / \mathrm{dL})\end{array}$} & $\mathrm{P}$ & $12.1 \pm 0.7$ & $10.9 \pm 0.4$ & $11.7 \pm 0.5$ & $10.4 \pm 0.6$ & $13.3 \pm 2.3$ & $11.4 \pm 0.5$ \\
\hline & B & $9.3 \pm 0.3$ & $10.4 \pm 0.4$ & $9.6 \pm 0.2$ & $11.4 \pm 0.4$ & $9.3 \pm 0.6$ & $9.8 \pm 0.2$ \\
\hline \multirow{2}{*}{$\begin{array}{l}\text { Total protein } \\
(\mathrm{g} / \mathrm{dL})\end{array}$} & $\mathrm{P}$ & $5.8 \pm 0.1 *$ & $5.6 \pm 0.1$ & $5.7 \pm 0.1$ & $5.5 \pm 0.2$ & $6.0 \pm 0.2$ & $5.7 \pm 0.1$ \\
\hline & B & $6.0 \pm 0.1$ & $5.9 \pm 0.1$ & $6.0 \pm 0.0$ & $6.1 \pm 0.2$ & $6.0 \pm 0.1$ & $6.0 \pm 0.0$ \\
\hline \multirow{2}{*}{$\begin{array}{l}\text { Albumin/globulin } \\
\text { ratio }\end{array}$} & $\mathrm{P}$ & $1.04 \pm 0.03$ & $1.08 \pm 0.04$ & $1.05 \pm 0.03$ & $1.11 \pm 0.10$ & $0.91 \pm 0.08 *$ & $1.08 \pm 0.02$ \\
\hline & B & $1.15 \pm 0.03$ & $1.17 \pm 0.03$ & $1.16 \pm 0.02$ & $1.14 \pm 0.08$ & $1.07 \pm 0.03$ & $1.17 \pm 0.02$ \\
\hline \multirow{2}{*}{$\begin{array}{l}\text { Albumin } \\
(\mathrm{g} / \mathrm{dL})\end{array}$} & $\mathrm{P}$ & $2.90 \pm 0.04$ & $2.87 \pm 0.05$ & $2.89 \pm 0.03$ & $2.87 \pm 0.10$ & $2.76 \pm 0.08$ & $2.91 \pm 0.04$ \\
\hline & B & $3.19 \pm 0.03$ & $3.16 \pm 0.04$ & $3.18 \pm 0.03$ & $3.25 \pm 0.09$ & $3.11 \pm 0.06$ & $3.19 \pm 0.03$ \\
\hline \multirow{2}{*}{$\begin{array}{l}\text { Alpha globulin } \\
(\mathrm{g} / \mathrm{dL})\end{array}$} & $\mathrm{P}$ & $1.06 \pm 0.02$ & $1.07 \pm 0.03$ & $1.06 \pm 0.02$ & $1.11 \pm 0.07$ & $1.15 \pm 0.08$ & $1.06 \pm 0.02$ \\
\hline & $\mathrm{B}$ & $0.97 \pm 0.02$ & $0.99 \pm 0.02$ & $0.97 \pm 0.02$ & $1.04 \pm 0.05$ & $0.99 \pm 0.03$ & $0.97 \pm 0.02$ \\
\hline \multirow{2}{*}{$\begin{array}{l}\text { Beta globulin } \\
(\mathrm{g} / \mathrm{dL})\end{array}$} & $\mathrm{P}$ & $1.00 \pm 0.03$ & $0.99 \pm 0.03$ & $1.00 \pm 0.02$ & $0.91 \pm 0.06$ & $1.05 \pm 0.07$ & $0.99 \pm 0.02$ \\
\hline & B & $1.01 \pm 0.02$ & $1.01 \pm 0.02$ & $1.01 \pm 0.01$ & $1.04 \pm 0.05$ & $1.01 \pm 0.05$ & $1.01 \pm 0.01$ \\
\hline \multirow{2}{*}{$\begin{array}{l}\text { Gamma globulin } \\
(\mathrm{g} / \mathrm{dL})\end{array}$} & $\mathrm{P}$ & $0.81 \pm 0.05^{*}$ & $0.65 \pm 0.06$ & $0.76 \pm 0.04$ & $0.63 \pm 0.12$ & $0.98 \pm 0.15^{*}$ & $0.72 \pm 0.04$ \\
\hline & B & $0.83 \pm 0.03 *$ & $0.75 \pm 0.04$ & $0.80 \pm 0.03$ & $0.79 \pm 0.07$ & $0.92 \pm 0.09$ & $0.78 \pm 0.03$ \\
\hline
\end{tabular}

H. somni-Histophilus somni; P. multocida - Pasteurella multocida; M. haemolytica - Mannheimia haemolytica; $\mathrm{P}-$ at the time of primary immunisation; $\mathrm{B}$ - at the time of booster immunisation; * - significant difference $(\mathrm{P}<0.05)$ to the negative

\begin{tabular}{|c|c|c|c|c|c|c|c|}
\hline \multirow{2}{*}{ (b) } & & \multicolumn{2}{|c|}{ H. somni } & \multicolumn{2}{|c|}{ P. multocida } & \multicolumn{2}{|c|}{ M. haemolytica } \\
\hline & & Positive & Negative & Positive & Negative & Positive & Negative \\
\hline \multirow{2}{*}{$\begin{array}{l}\text { d-ROMs } \\
\text { (Carr U) }\end{array}$} & $\mathrm{P}$ & $77.2 \pm 2.8$ & $83.4 \pm 2.7$ & $80.3 \pm 2.2$ & $78.4 \pm 3.4$ & $77.0 \pm 5.7$ & $80.8 \pm 2.1$ \\
\hline & $\mathrm{B}$ & $76.0 \pm 1.7$ & $78.1 \pm 2.5$ & $76.7 \pm 1.6$ & $79.4 \pm 5.1$ & $74.8 \pm 2.9$ & $77.5 \pm 1.7$ \\
\hline \multirow{2}{*}{$\begin{array}{l}\text { BAP } \\
(\mu \mathrm{M})\end{array}$} & $\mathrm{P}$ & $2,934 \pm 50$ & $2,985 \pm 52$ & $2,959 \pm 38$ & $2,951 \pm 114$ & $2,862 \pm 83$ & $2,978 \pm 40$ \\
\hline & $\mathrm{B}$ & $2,843 \pm 39$ & $2,802 \pm 52$ & $2,825 \pm 33$ & $2,816 \pm 122$ & $2,933 \pm 85$ & $2,802 \pm 33$ \\
\hline \multirow{2}{*}{ OSI } & $\mathrm{P}$ & $2.64 \pm 0.10$ & $2.81 \pm 0.10$ & $2.73 \pm 0.08$ & $2.66 \pm 0.04$ & $2.69 \pm 0.19$ & $2.73 \pm 0.08$ \\
\hline & $\mathrm{B}$ & $2.69 \pm 0.08$ & $2.82 \pm 0.11$ & $2.74 \pm 0.07$ & $2.85 \pm 0.24$ & $2.57 \pm 0.15$ & $2.79 \pm 0.07$ \\
\hline
\end{tabular}

H. somni-Histophilus somni; P. multocida - Pasteurella multocida; M. haemolytica - Mannheimia haemolytica; $\mathrm{P}-$ at the time of primary immunisation; B - at the time of booster immunisation; d-ROMs - diacron reactive oxygen metabolites; Carr U - Carratelli units; BAP - biological antioxidant potential; OSI - oxidative stress index 
Table 3. Pearson's correlation analysis

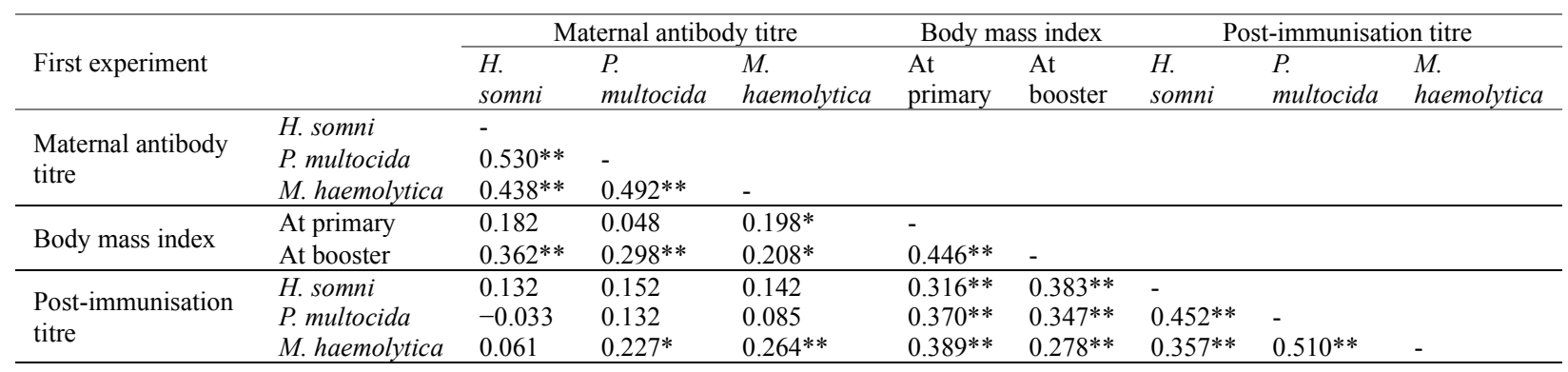

H. somni - Histophilus somni; P. multocida - Pasteurella multocida; M. haemolytica - Mannheimia haemolytica; Maternal antibody titre - the titre at the primary immunisation; Post-immunisation titre - the titre at three weeks after booster immunisation; * $-\mathrm{P}<0.05 ; * *-\mathrm{P}<0.01$

\begin{tabular}{lccc}
\hline \multirow{2}{*}{ Second experiment } & \multicolumn{3}{c}{ Maternal antibody titre } \\
\cline { 2 - 3 } & H. somni & P. multocida & -0.093 \\
\hline Total cholesterol & -0.171 & 0.101 & -0.043 \\
Blood urea nitrogen & 0.142 & $0.539^{* *}$ & $0.306^{*}$ \\
Total protein & $0.593 * *$ & $-0.485^{* *}$ & $-0.418^{* *}$ \\
Albumin/globulin ratio & $-0.610^{* *}$ & -0.009 & -0.119 \\
Albumin & -0.068 & 0.092 & 0.000 \\
Alpha globulin & 0.003 & $0.303^{*}$ & 0.158 \\
Beta globulin & $0.485^{* *}$ & $0.683^{* *}$ & $0.512^{* *}$ \\
Gamma globulin & $0.766^{* *}$ & \\
\hline
\end{tabular}

H. somni - Histophilus somni; P. multocida - Pasteurella multocida; M. haemolytica - Mannheimia haemolytica; Maternal antibody titre - the titre at the primary immunisation; * $-\mathrm{P}<0.05 ; * *-\mathrm{P}<0.01$
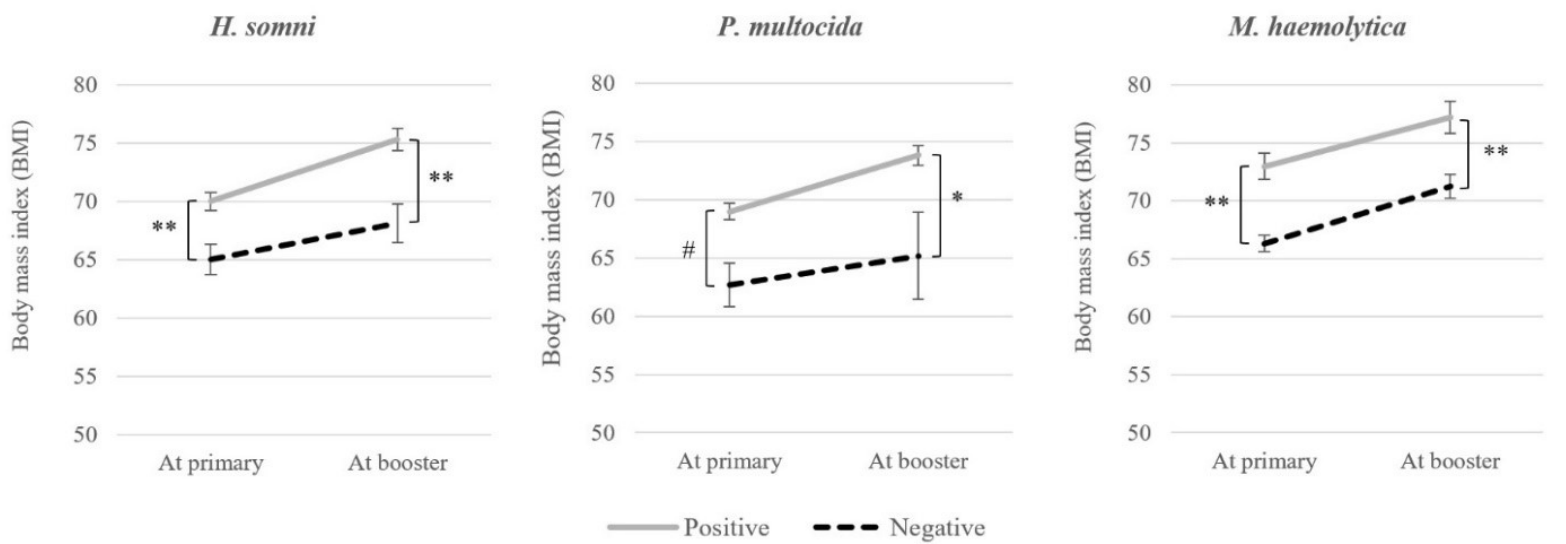

Fig. 1. BMI of the positive and negative group calves at the primary and booster immunisation in the first experiment. * $-\mathrm{P}<0.05 ; * *-\mathrm{P}<0.01 ; \#-\mathrm{P}=0.062$

Maternal titres against $H$. somni and M. haemolytica for the respective positive calves were significantly higher than those for the negative calves $(0.118 \pm 0.013$ vs. $0.096 \pm 0.017 ; \mathrm{P}<0.01$ and $255 \pm 30.1$ vs. $145 \pm 12.2$; $\mathrm{P}<0.01$ ). Although the maternal titre against $P$. multocida for the $P$. multocida-positive calves was higher than for the negative calves, this was not significant $(105 \pm 7.1$ vs. $83 \pm 24.7 ; \mathrm{P}=0.268$ ). The $\mathrm{BMI}$ at both the primary and booster immunisations of the $H$. somni- or $M$. haemolytica-positive calves was significantly higher than that for the negative calves $(\mathrm{P}<0.01)$. Similarly, the BMI of the P. multocida-positive calves was higher than that of the negative calves at the primary $(\mathrm{P}=0.062)$ and booster immunisations $(\mathrm{P}<0.05)$ (Fig. 1).

Table 2 presents nutritional status and OS marker values of the positive and negative calves in the second and third experiments. Serum total protein at the primary immunisation and gamma globulin at each immunisation of the H. somni-positive calves were higher than those of the negative calves $(\mathrm{P}<0.05)$. Serum gamma globulin at the primary immunisation of the M. haemolyticapositive calves was higher than that of the negative calves $(\mathrm{P}<0.05)$. Additionally, the serum $\mathrm{A} / \mathrm{G}$ ratio at primary immunisation of the $M$. haemolytica-negative group was higher than that of the positive group $(\mathrm{P}<0.05)$. No significant differences were observed in serum d-ROMs, BAP or OSI for each of the three bacteria (Table 2).

Table 3 presents the relationships between maternal antibody titre at the primary immunisation, BMI at both immunisations and antibody titre after immunisations (first experiment) and between maternal antibody titre and nutritional status at the time of primary immunisation (second experiment). In the first experiment, no strong or moderate correlation was found between maternal antibody titre, BMI and the titre after immunisations. In the second experiment, serum gamma globulin levels were strongly related to maternal titres against $H$. somni $(\mathrm{r}=0.766, \mathrm{P}<0.01)$ or P. multocida $(\mathrm{r}=0.683, \mathrm{P}<0.01)$ and moderately related to titres against $M$. haemolytica $(\mathrm{r}=0.512, \mathrm{P}<0.01)$. Serum total protein was moderately 
related to maternal titres against $H$. somni $(\mathrm{r}=0.593$, $\mathrm{P}<0.01)$ and $P$. multocida $(\mathrm{r}=0.539, \mathrm{P}<0.01)$ but only weakly related to titres against $M$. haemolytica $(\mathrm{r}=0.306$, $\mathrm{P}<0.05)$. There were negative correlations between

(1)

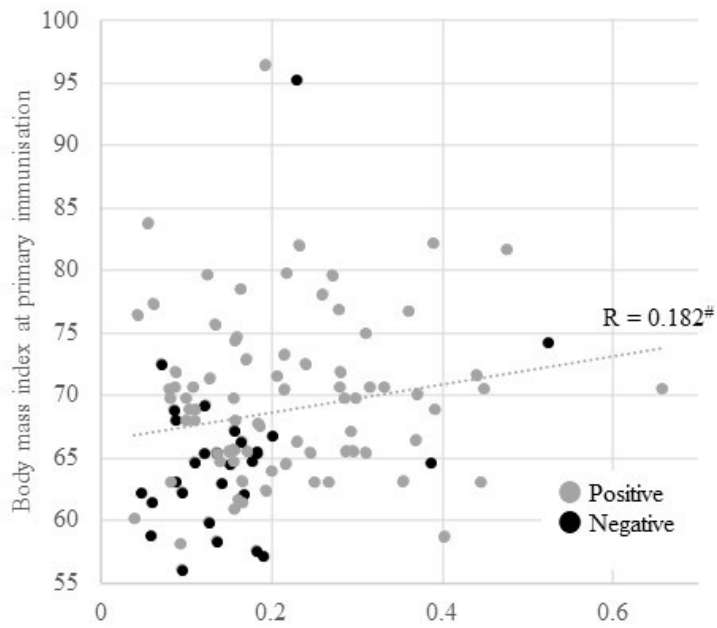

Antibody titre at primary immunisation for $\mathrm{H}$. somni

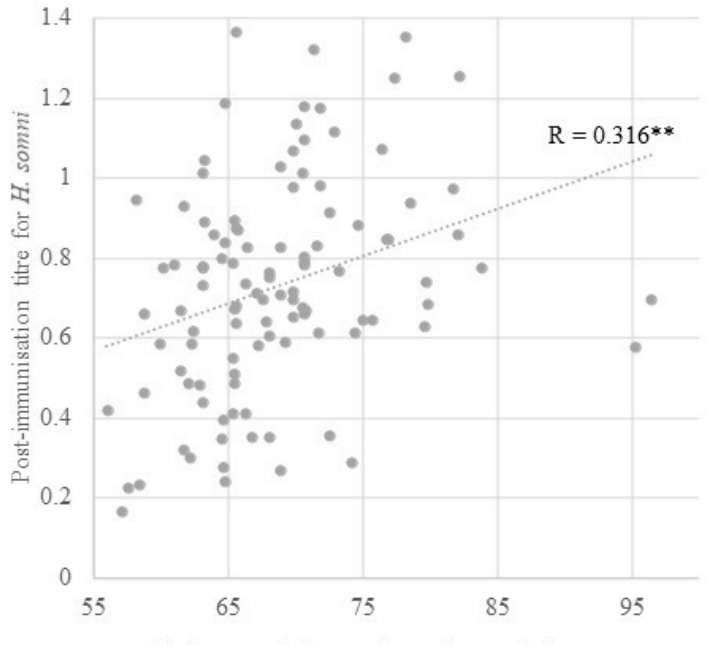

Body mass index at primary immunisation

(2)

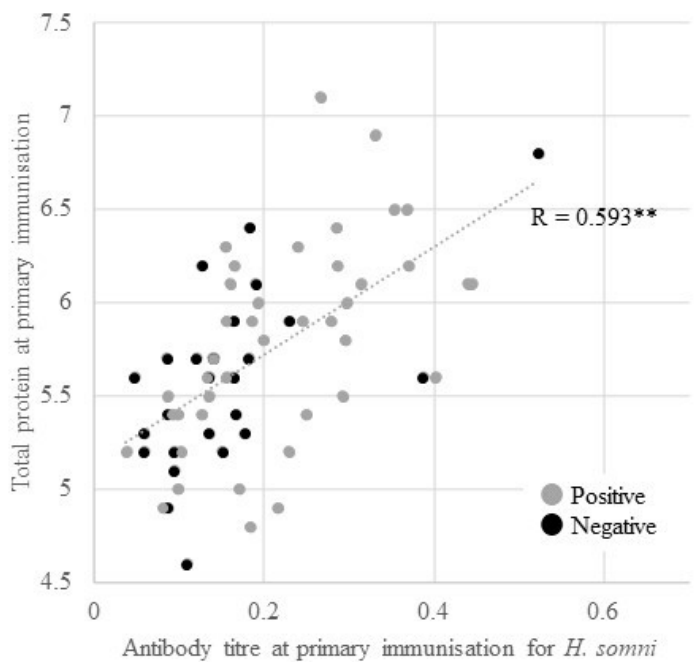

the $\mathrm{A} / \mathrm{G}$ ratio and maternal titres against $H$. somni, $P$. multocida and $M$. haemolytica $(\mathrm{r}=-0.610, \mathrm{P}<0.01$; $\mathrm{r}=-0.485, \mathrm{P}<0.01 ;$ and $\mathrm{r}=-0.418, \mathrm{P}<0.01$, respectively) (Table 3, Fig. 2).
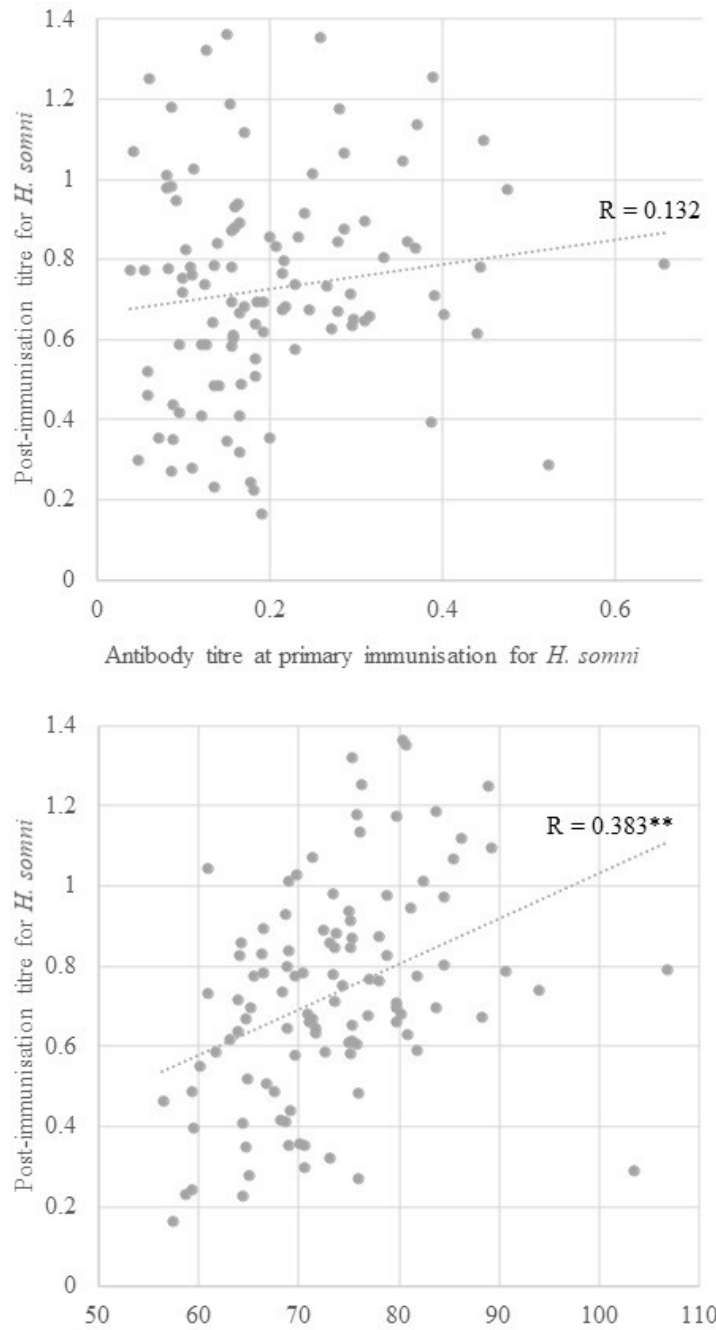

Body mass index at booster immunisation

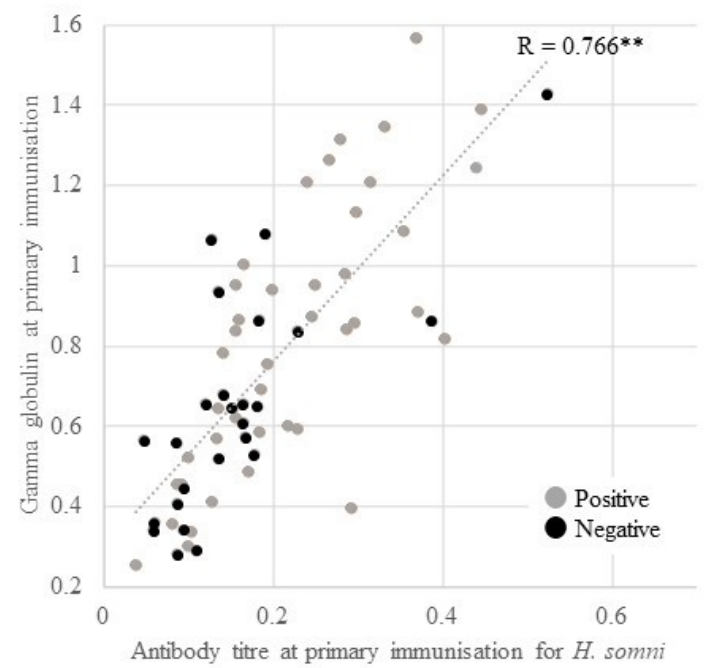

Fig. 2. Relationship between body mass index at primary immunisation and maternal antibody titre against Histophilus somni at primary immunisation; post-immunisation titre and maternal antibody titre at primary immunisation against $H$. somni; post-immunisation titre against $H$. somni and body mass index at primary immunisation; and post-immunisation titre against $H$. somni and body mass index at booster vaccination in the first experiment (1) and between maternal antibody titre and serum total protein or gamma globulin at the primary immunisation in the second experiment (2). **- $\mathrm{P}<0.01 ; \#-\mathrm{P}<0.10$ 


\section{Discussion}

The results of our study suggest that calves with elevated MAL, increased serum total protein, elevated serum gamma globulin levels and ideal body weight exhibit positive antibody responses. These four factors were previously found to be strongly associated with the health status of calves $(6,8,17,18)$. It has already been reported that a significant reduction in the incidence of respiratory disease as well as higher antibody titres in responses detected using the same antigen were observed in calves immunised with the same vaccine as the one used in this study (16). Therefore, early immunisation probably mitigates BRD in young calves, and the health status of calves at immunisation is very important for preventing BRD.

The results of the first experiment indicate that maternal antibody titres at the primary immunisation were higher for calves with a positive response to immunisation than for calves with a negative response. This finding is in contrast to that made by Foote et al. (7) that maternal antibodies lower the effectiveness of the calf immune response to immunisation. In stimulating an adaptive immune response, vaccination involves B cell memory. These cells have been found to be activated in neonatal calves, even in the presence of maternally transferred immunoglobulin $\mathrm{G}(\mathrm{IgG})(7,25)$, and $\mathrm{IgG}$ is the major class of immunoglobulin present in ruminant colostrum (26). Kang et al. (12) suggested that an early antibody response with $\mathrm{IgG}$ immune complexes contributes to the optimal formation of B cell memory. For these reasons, we presume that maternal antibodies probably do not inhibit but may assist the B cell memory responses and increase antibody titres following the administration of booster doses of inactivated vaccines.

In addition, the results of the second experiment demonstrated that higher serum total protein and gamma globulin levels were positively associated with early immunisation. Donovan et al. (6) noted that a lower serum total protein concentration was a significant risk factor for the occurrence and severity of pneumonia. Furman-Fratczak et al. (8) suggested that calves with elevated gamma globulin had fewer respiratory tract infections. Serum total protein and gamma globulin levels are strongly associated with the calves' defence against infectious diseases, and it has been reported that serum total protein and serum gamma globulin concentration correlate with $\operatorname{IgG}$ concentration $(3,30)$. This experiment also revealed a strong correlation between maternal antibody titres and serum total protein or gamma globulin. Initially, calves acquire immunity through absorption of antibodies found in the colostrum of their dams (13). It has been previously demonstrated that the failure of passive transfer of immunity in calves was influenced primarily by the quality of colostrum, amount of colostrum ingested and time between birth and first feeding (22). In this study, although each calf was fed adequate amounts of a colostrum replacer, the time between birth and first feeding might not have been uniform. This implies that it may be necessary to provide adequate amounts of good-quality colostrum early in life to ensure a successful response to immunisation in young calves.

The first experiment also revealed that calves with a lower BMI had a lower antibody response to immunisation. BMI is widely used as an indicator of underweight or normal weight in humans (29). Ohtsuka et al. (18) revealed that suckling calves with low body weight had lower levels of peripheral blood mononuclear cell mRNA for interleukin 4, the interleukin which induces the differentiation of naive CD4 $\mathrm{T}$ cells into type 2 helper $\mathrm{T}$ cells, drives the immunoglobulin class switch to IgG1 and IgE in B cells and induces alternative macrophage activation in macrophages (10). Type 2 helper $\mathrm{T}$ cells drive the type-2 pathway, induce humoral immunity and upregulate antibody production against extracellular organisms (14). Moreover, Olofin et al. (19) suggested that underweight and poor growth were associated with higher mortality in human children less than 5 years of age through secondary immune suppression and increased susceptibility to infections. Based on the above findings, we concluded that underweight calves have less ability to respond to vaccine antigens. In addition, this experiment also revealed only weak or slight correlations in maternal antibody titres at primary immunisation, BMI and post-immunisation titres. This suggests that BMI and maternal antibodies may independently affect antibody responses.

In the second experiment, there was no difference in serum T-cho and albumin levels between the two groups of calves. Zhang et al. (31) showed that serum $\mathrm{T}$-cho and albumin are useful biochemical indicators of malnutrition. Ohtsuka et al. (17) reported that calves experimentally provided with insufficient nutrition have a low number of immune cells, with a low value of serum T-cho. However, Rytter et al. (23) revealed that responses to immunisation are reduced in severely malnourished human children, but not in those with moderate malnutrition. We believe that malnourishment in calves on commercial dairy farms severe enough to lead to attenuation of their response to immunisation is unlikely and that a ubiquitous adequate nutrition status is the reason why no relationship was observed between the antibody response and serum T-cho and albumin levels in this experiment.

Furthermore, markers of OS also did not differ significantly between the two groups in the third experiment. Sordillo et al. (24) reported that OS diminishes the functional capabilities of immune cells and increases an animal's susceptibility to disease. Generally, it has been presumed that OS is associated with the bovine immune system $(2,5)$. The immune responses in young calves are weaker than those in adult cattle (11), but although we cannot deny that OS affects antibody responses to early immunisation, it is assumed that MAL and BMI influence a young calf's antibody response to early immunisation more strongly than OS. 
In conclusion, MAL and satisfactory BMI were positively correlated with antibody production, following booster immunisation with inactivated vaccines in young Holstein calves. The present results suggest the possibility that suitable colostrum feedings are fundamental to successful early immunisation with inactivated vaccines in young calves.

Conflict of Interests Statement: The authors declare that there is no conflict of interests regarding the publication of this article.

Financial Disclosure Statement: This research did not receive any specific grant from funding agencies of the public, commercial or not-for-profit sectors.

Animal Rights Statement: All animals were regular patients of the Farm Animal Clinical Services located at the Rakuno Gakuen University Animal Medical Center. All procedures and animal care provided in the study were in accordance with the guidelines approved by the Rakuno Gakuen University Animal Experiment and Care Committee.

Acknowledgements: We thank Dr. Shuichi Kubota for his support of this study and thank his employer, Kyoto Biken Laboratories, Inc. for providing the vaccines and measuring serum antibody levels free of charge. We also appreciate the cooperation of the farm owner and the personnel who were involved in this study. Lastly, we would like to thank Enago (www.enago.jp) for the English language review.

\section{References}

1. Abuelo A., Hernández J., Benedito J.L., Castillo C.: Oxidative stress index $(\mathrm{OSi})$ as a new tool to assess redox status in dairy cattle during the transition period. Animal 2013, 7, 1374-1378, doi: $10.1017 /$ S1751731113000396.

2. Abuelo A., Hernández J., Benedito J.L., Castillo C.: Redox biology in transition periods of dairy cattle: role in the health of periparturient and neonatal animals. Antioxidants (Basel) 2019, 8, 20, doi: 10.3390/antiox 8010020 .

3. Alves A.C., Alves N.G., Ascari I.J., Junqueira F.B., Coutinho A.S., Lima R.R., Pérez J.R.O., de Paula S.O., Furusho-Garcia I.F., Abreu L.R.: Colostrum composition of Santa Inês sheep and passive transfer of immunity to lambs. J Dairy Sci 2015, 98, 3706-3716, doi: 10.3168/jds.2014-7992.

4. Benzie I.F.F., Strain J.J.: The ferric reducing ability of plasma (FRAP) as a measure of "antioxidant power": the FRAP assay. Anal Biochem 1996, 239, 70-76, doi: 10.1006/abio.1996.0292.

5. Celi P.: Biomarkers of oxidative stress in ruminant medicine. Immunopharmacol Immunotoxicol 2011, 33, 233-240, doi: 10.3109/08923973.2010.514917.

6. Donovan G.A., Dohoo I.R., Montgomery D.M., Bennett F.L.: Associations between passive immunity and morbidity and mortality in dairy heifers in Florida, USA. Prev Vet Med 1998, 34, 31-46, doi: 10.1016/s0167-5877(97)00060-3.

7. Foote M.R., Nonnecke B.J., Beitz D.C., Waters W.R.: AntigenSpecific B-Cell responses by neonatal calves after early vaccination. J Dairy Sci 2007, 90, 5208-5217, doi: $10.3168 /$ jds.2007-0285.
8. Furman-Fratczak K., Rzasa A., Stefaniak T.: The influence of colostral immunoglobulin concentration in heifer calves' serum on their health and growth. J Dairy Sci 2011, 94, 5536-5543, doi: 10.3168/jds.2010-3253.

9. Hodgins D.C., Shewen P.E.: Serologic responses of young colostrum fed dairy calves to antigens of Pasteurella haemolytica A1. Vaccine 1998, 16, 2018-2025, doi: 10.1016/s0264410x(98)00086-3.

10. Junttila I.S.: Tuning the cytokine responses: an update on interleukin (IL)-4 and IL-13 receptor complexes. Front Immunol 2018, 9, 888, doi: 10.3389/fimmu.2018.00888.

11. Kampen A.H., Olsen I., Tollersrud T., Storset A.K., Lund A.: Lymphocyte subpopulations and neutrophil function in calves during the first 6 months of life. Vet Immunol Immunopathol 2006, 113, 53-63, doi: 10.1016/j.vetimm.2006.04.001.

12. Kang S., Keener A.B., Jones S.Z., Benschop R.J., Caro-Maldonado A., Rathmell J.C., Clarke S.H., Matsushima G.K., Whitmire J.K., Vilen B.J.: IgG-immune complexes promote B cell memory by inducing BAFF. J Immunol 2016, 196, 196-206, doi: 10.4049/jimmunol.1402527.

13. Kertz A.F., Hill T.M., Quigley J.D., Heinrichs A.J., Linn J.G., Drackley J.K.: A 100-year review: calf nutrition and management. J Dairy Sci 2017, 100, 10151-10172, doi: 10.3168/jds.201713062.

14. Kidd P.: Th1/Th2 balance: the hypothesis, its limitations, and implications for health and disease. Altern Med Rev 2003, 8, 223-246.

15. Mori K., Kato T., Yokota O., Ohtsuka H.: Field trial of primary and booster dose of inactivated vaccine against bovine respiratory bacteria in young Holstein calves. J Vet Res 2020, 64, 223-230, doi: 10.2478 jvetres-2020-0042.

16. Nagai K., Otomaru K., Ogawa R., Oishi S., Wataya K., Honkawa Y., Iwamoto Y., Ando T., Hyakutake K., Shirahama H., Habiby G., Kubota C.: Effect of combined vaccination for Pasteurella multocida, Mannheimia haemolytica, and Histophilus somni to prevent respiratory diseases in young Japanese Black calves in the field. J Vet Med Sci 2019, 81, 1355-1358, doi: 10.1292/jvms.190256.

17. Ohtsuka H., Fukunaga N., Fukuda S., Hatsugaya A., Hayashi T., Hara H., Koiwa M., Abe R., Kawamura S.: Effect of nutritional conditions on changes in leukocyte populations in Japanese Black Calves. J Vet Med Sci 2005, 67, 183-185, doi: 10.1292/jvms.67.183.

18. Ohtsuka H., Kishiki F., Ando T., Mukai M., Kohiruimaki M., Tanami E.: Relationship between growth condition and endocrine or immune condition in healthy suckling Holstein calves. J Jpn Vet Med Assoc 2011, 64, 294-299, doi: 10.12935/jvma.64.294.

19. Olofin I., McDonald C.M., Ezzati M., Flaxman S., Black R.E., Fawzi W.W., Caulfield L.E., Danaei G.: Associations of suboptimal growth with all-cause and cause-specific mortality in children under five years: a pooled analysis of ten prospective studies. PLoS One 2013, 8, e64636, doi: 10.1371/journal.pone.0064636.

20. Otomaru K., Kubota S., Ohtsuka H., Ando T., Koiwa M.: Field application of mixed vaccine including inactivated Pasteurella multocida, Mannhemia haemolytica and Histophilus somni to Japanese Black calves for preventing development of respiratory disease. J Jpn Vet Med Assoc 2012, 65, 767-770, doi: 10.12935/jvma.65.767.

21. Otomaru K., Ogawa R., Oishi S., Iwamoto Y., Hong H., Nagai K., Hyakutake K., Kubota C., Kaneshige T.: Effect of beta-carotene supplementation on the serum oxidative stress biomarker and antibody titer against live bovine respiratory syncytial virus vaccination in Japanese black calves. Vet Sci 2018, 5, 102, doi: 10.3390/vetsci5040102.

22. Reschke C., Schelling E., Michel A., Remy-Wohlfender F., Meylan M.: Factors associated with colostrum quality and effects on serum gamma globulin concentrations of calves in Swiss dairy herds. J Vet Intern Med 2017, 31, 1563-1571, doi: 10.1111/jvim. 14806.

23. Rytter M.J.H., Kolte L., Briend A., Friis H., Christensen V.B.: The immune system in children with malnutrition - A systematic review. PLoS One 2014, 9, e105017, doi: 10.1371/journal.pone.0105017. 
24. Sordillo L.M., Aitken S.L.: Impact of oxidative stress on the health and immune function of dairy cattle. Vet Immunol Immunopathol 2009, 128, 104-109, doi: 10.1016/j.vetimm.2008.10.305.

25. Stabel J.R., Bannantine J.P., Eda S., Robbe-Austerman S.: Induction of $\mathrm{B}$ cell responses upon experimental infection of neonatal calves with Mycobacterium avium subsp. paratuberculosis. Clin Vaccine Immunol 2011, 18, 1139-1149, doi: 10.1128/CVI.00058-11.

26. Stelwagen K., Carpenter E., Haigh B., Hodgkinson A., Wheeler T.T.: Immune components of bovine colostrum and milk. J Anim Sci 2009, 87, 3-9, doi: 10.2527/jas.2008-1377.

27. Tucci P., Estevez V., Becco L., Cabrera-Cabrera F., Grotiuz G., Reolon E., Marín M.: Identification of leukotoxin and other vaccine candidate proteins in a Mannheimia haemolytica commercial antigen. Heliyon 2016, 2, e00158, doi: 10.1016/j.heliyon.2016.e00158.

28. United States Department of Agriculture, Animal and Plant Health Inspection Service, Veterinary Service, Center for Epidemiology and Animal Health, National Animal Health Monitoring Service: Health and management practices on U.S. Dairy Operations, 2014. U.S.D.A., Fort Collins, 2018

29. Weir C., Jan A.: BMI classification percentile and cut off points. In: StatPearls, StatPearls Publishing, Treasure Island 2021, https:/www.ncbi.nlm.nih.gov/books/NBK541070.

30. Wilm J., Costa J.H.C., Neave H.W., Weary D.M., von Keyserlingk M.A.G.: Technical note: serum total protein and immunoglobulin $\mathrm{G}$ concentrations in neonatal dairy calves over the first 10 days of age. J Dairy Sci 2018, 101, 6430-6436, doi: 10.3168/jds.2017-13553.

31. Zhang Z., Pereira S.L., Luo M., Matheson E.M.: Evaluation of blood biomarkers associated with risk of malnutrition in older adults: A systematic review and meta-analysis. Nutrients 2017, 9, 829 doi: $10.3390 /$ nu9080829. 